\title{
Lattice dynamics and thermophysical properties of h.c.p. Os and Ru from the quasi-harmonic approximation
}

\author{
Mauro Palumbo [1], Andrea Dal Corso [1,2] \\ [1] International School for Advanced Studies (SISSA), Trieste, ITALY \\ [2] CNR-IOM, Trieste, ITALY
}

July 10, 2018

\begin{abstract}
We report first-principles phonon frequencies and anharmonic thermodynamic properties of h.c.p. Os and $\mathrm{Ru}$ calculated within the quasi-harmonic approximation, including Grüneisen parameters, temperature-dependent lattice parameters, thermal expansion, and isobaric heat capacity. We discuss the differences between a full treatment of anisotropy and a simplified approach with a constant $c / a$ ratio. The results are systematically compared with the available experimental data and an overall satisfactory agreement is obtained.
\end{abstract}




\section{Introduction}

Osmium (Os) and Ruthenium (Ru), both belonging to the same group (VIII B) of the periodic table, are hexagonal elements with high melting points, densities, and cohesive energies. The former has the highest measured density among the elements [1, 2], it is very hard and brittle even at high temperatures, and it is used in Pt and Ir alloys to enhance their mechanical properties and in the chemical industry as osmium tetroxide. Ruthenium finds use in electronic industry for manufacturing electrical contacts and chip resistors as well as in chemical industry for use as anodes for chlorine production in electrochemical cells.

The experimental determination of phonon dispersions and thermophysical properties of both elements is difficult. Only a few phonon frequencies have been measured for Os using Raman spectroscopy [3, 4], while for Ru phonon dispersions have been obtained recently using inelastic neutron scattering [5]. A previous work also reports experimental phonon frequencies along the [001] direction [6]. Available crystallographic data on Os have been reviewed by Arblaster [7], who reports assessed values of the lattice parameters and thermal expansion coefficients from 0 to $1300 \mathrm{~K}$ and references to prior experimental work [8, 9, 10]. The available thermodynamic properties of $\mathrm{Ru}$ are reported in [11] and references therein. Arblaster [11, 12] also reports assessed values on thermodynamic quantities and crystallographic properties of $\mathrm{Ru}$. Additional experimental work can be found in Refs. [8, 13, 14, 9, 10].

Several theoretical works have been dedicated to h.c.p. Os [15, 16, 17, 18, 19]. An experimental investigation using the diamond anvil cell [20] found that the bulk modulus of Os was $462 \mathrm{GPa}$, higher than diamond, but later studies reported lower values [21, 22]. The most recent theoretical studies have focused on the high-pressure behavior of this element where an anomaly in the first derivative of the $c / a$ ratio with respect to pressure has been found experimentally at $25 \mathrm{GPa}$ [22]. This anomaly was later disproven by Dubrovinsky et al. [16], who report a detailed experimental and theoretical investigation of Os behavior at very high pressure (up to $770 \mathrm{GPa}$ ) using state-of-the-art methods, including dynamical mean field theory (DMFT) and spin-orbit coupling, but without computing phonons. They found anomalies at approximately $150 \mathrm{GPa}$ and $440 \mathrm{GPa}$. Temperature-dependent properties have been calculated by Liu et al. [18] and Deng et al. [17]. The former used a quasi-harmonic Debye model, where the temperature-dependence of the free energy is obtained from a volume-dependent Debye temperature and not from phonon frequencies. On the contrary, Deng et al. performed phonon calculations for different volumes and then applied a Birch equation of state (EOS) to derive quasi-harmonic quantities. The phonon spectra of $\mathrm{Ru}$ has been theoretically investigated by Heid et. al. [5] and Souvatzis et al. [23]. The latter authors have also applied a quasi-harmonic approach, including the evaluation of the thermal expansion, similarly to Yaozhuang et al. [24]. We remark that anomalies found by Dubrovinsky et al. occur at pressures well above the range of quasi-harmonic calculations.

With the exception of the work by Souvatzis et al., however, all previous theoretical quasi-harmonic results rely on ultrasoft pseudopotentials or earlier-developed methods. Published works reporting results at the quasi-harmonic level use only one of the well-established LDA/PBE functionals without an extensive comparison with other functionals, previous theoretical results and experimental data. Besides, some properties such as mode Grüneisen parameters and the temperature dependence of 
both $a$ and $c$ lattice parameters were not reported in the literature and anisotropic linear thermal expansions were reported only for $\mathrm{Ru}$.

In this paper we report anharmonic results calculated with the quasi-harmonic approximation using PAW pseudopotentials from the Quantum Espresso pslibrary1.0 [25, 26. We test three different functionals (LDA, PBE and PBEsol) at the same level of theory. We extensively compare our results with an extended set of experimental data and previous theoretical results aiming at providing a consistent set of reliable theoretical data within the quasi-harmonic approximation. Finally we investigate the possibility that also $\mathrm{Os}$ and $\mathrm{Ru}$ have anomalous features of the phonon dispersions as those we found recently for Re and Tc [27], i.e. the presence of soft modes at the $\Gamma$ and $\mathrm{H}$ points in the Brillouin zone. Anomalies were also found in the Grüneisen parameters of Re and Tc and are studied here for Os and Ru.

Phonon dispersions were computed for several volumes and lattice parameters. From the phonon frequencies, the Grüneisen parameters, the thermal expansion and other thermophysical quantities as a function of temperature were derived. The results were first calculated assuming a constant $c / a$ ratio and computing volume dependent phonon frequencies. A full treatment of anisotropy in the hexagonal cell was then applied to obtain the temperature dependence of $a$ and $c$ and the thermal expansion tensor.

\section{Computational method}

All calculations in the present work were carried out using Density Functional Theory (DFT) and plane waves basis sets as implemented in the Quantum Espresso (QE) package [28, 29]. The thermo_pw package [30] was used for obtaining harmonic and quasi-harmonic properties. The LDA [31], the PBE-GGA [32] and the revised PBE functional for densely-packed solids (PBEsol) [33] were tested for the exchangecorrelation functional. We employed the Os.pz-spn-kjpaw_psl.1.0.0.UPF, Os.pbe-spnkjpaw_psl.1.0.0.UPF, Os.pbesol-spn-kjpaw_psl.1.0.0.UPF, Ru.pz-spn-kjpaw_psl.1.0.0.UPF, Ru.pbe-spn-kjpaw_psl.1.0.0.UPF and Ru.pbesol-spn-kjpaw_psl.1.0.0.UPF pseudopotentials from the pslibrary 1.0 [25, 26].

We have chosen computational settings to ensure that all investigated properties and in particular phonon frequencies are well converged. The kinetic energy cut-off was set to 50 Ry while the charge density cut-off was set to 200 Ry, except for Os PBE and PBEsol for which it was set to 300 Ry. The integration over the Brillouin Zone (BZ) was performed employing a $18 \times 18 \times 12$ Monkhorst-Pack $\vec{k}$-points mesh and a Methfessel-Paxton smearing scheme with 0.02 Ry width. The Density Functional Perturbation Theory (DFPT) as implemented in the phonon code [34, 35] was used to calculate phonon frequencies on a $6 \times 6 \times 4$ grid of $\vec{q}$-points and a Fourier interpolation was used for other points in the BZ.

In the quasi-harmonic approximation [36], the Helmholtz energy of a crystalline solid is

$$
F(T, X)=U_{0}(X)+F^{\mathrm{vib}}(T, X)+F^{\mathrm{el}}(T, X)
$$

where $U_{0}$ is the static energy at $0 \mathrm{~K}, F^{\mathrm{vib}}$ the contribution due to lattice vibrations and $F^{\mathrm{el}}$ the energy due to electronic excitations. In the adiabatic approximation, each term is treated separately. $\mathrm{X}$ refers to any variable upon which the above energies may depend and most often it is the unit cell volume as for example for cubic lattices. In the 
case of an h.c.p. cell, $\mathrm{X}$ refers to the two lattice parameters $a$ and $c / a$, although it is common to assume that $c / a$ is constant as a function of temperature and thus consider the variation of $F$ only as a function of $a$ (or equivalently $V$ ). For a given $X$, the vibrational Helmholtz energy per cell is calculated as in the harmonic approximation:

$$
F^{\mathrm{vib}}(X, T)=\frac{1}{2 N} \sum_{\vec{q}, \nu} \hbar \omega(\vec{q}, \nu, X)+\frac{k_{\mathrm{B}} T}{N} \sum_{\vec{q}, \nu} \ln \left[1-\exp \left(\frac{-\hbar \omega(\vec{q}, \nu, X)}{k_{\mathrm{B}} T}\right)\right] .
$$

The first term on the right-hand side of the above equation is the Zero-Point Energy (ZPE), the second term is the phonon contribution at finite temperatures. The sums in Eq. (2) are taken over the phonon frequencies $\omega(\vec{q}, \nu, X)$, where $\nu$ denotes the different phonon branches and $\vec{q}$ the wave vectors within the first BZ. $k_{B}$ is the Boltzmann constant, $\hbar$ the reduced Planck constant, $T$ the absolute temperature, and $N$ the number of unit cells in the solid.

We carried out calculations both (a) assuming a constant $c / a$ and (b) evaluating the Helmholtz energy on a full grid $(a, c / a)$.

In the former case, we computed the phonon frequencies at 9 different volumes (or equivalently $a$ values), keeping the $c / a$ constant at the $0 \mathrm{~K}$ equilibrium value and fitting the resulting energies using a Murnaghan EOS [37]. The minimum $U_{0}(V)+F^{\mathrm{vib}}(T, V)$ at each temperature corresponds to zero pressure. In this way, the temperature dependence of the free energy $F$, the volume $V$ and the isothermal bulk modulus $B_{T}$ is directly obtained from the Murnaghan EOS. By numerical derivation of the volume we obtained the volume thermal expansion coefficient $\beta$ according to its definition

$$
\beta=\frac{1}{V}\left(\frac{\partial V}{\partial T}\right)_{P}
$$

The so-called Grüneisein mode parameters were calculated as

$$
\gamma_{\mathbf{q}, \nu}=-\frac{V}{\omega(\vec{q}, \nu, V)} \frac{\partial \omega(\vec{q}, \nu, V)}{\partial V}
$$

from the computed frequencies. The constant volume (isochoric) heat capacity was obtained from the phonon frequencies calculated as in the harmonic approximation

$$
C_{V}=\frac{k_{\mathrm{B}}}{N} \sum_{\vec{q}, \nu}\left(\frac{\hbar \omega(\vec{q}, \nu)}{k_{B} T}\right)^{2} \frac{\exp \left(\hbar \omega(\vec{q}, \nu) / k_{B} T\right)}{\left[\exp \left(\hbar \omega(\vec{q}, \nu) / k_{B} T\right)-1\right]^{2}},
$$

at each fixed volume in the quasi-harmonic grid. The isochoric heat capacity at each temperature is then obtained interpolating at the temperature-dependent volume values obtained at each temperature from the minimization of the free energy. Finally, the constant pressure (isobaric) heat capacity was obtained as [38]

$$
C_{P}=C_{V}+T V \beta^{2} B_{T}
$$

In the full anisotropic case (b), the calculations were carried out on a grid of points of $a$ and $c / a$ and then the Helmholtz energy at each $\mathrm{T}$ was fitted with quartic polynomials as a function of $a$ and $c / a$. For both Os and $\mathrm{Ru}$, a total of 25 grid points $(5 \times 5)$ were used with steps of 0.05 a.u. and 0.02 in $a$ and $c / a$, respectively. The grid was approximately centered at the equilibrium values of $a$ and $c / a$ at $0 \mathrm{~K}$. We derive the variation with temperature of $a$ and $c$ from the minimization of $F$. 
The components of the thermal expansion are obtained from the temperature variation of the lattice parameters as

$$
\begin{gathered}
\alpha_{1}=\alpha_{2}=\frac{1}{a(T)} \frac{d a(T)}{d T}, \\
\alpha_{3}=\frac{1}{c(T)} \frac{d c(T)}{d T},
\end{gathered}
$$

since in the present hexagonal case only two thermal expansion terms are independent. Finally, the volume thermal expansion $\beta$ is given by $\beta=2 \alpha_{1}+\alpha_{3}$.

The electronic contribution to the heat capacity was obtained in the single-particle approximation from the electronic DOS at the equilibrium lattice parameters at $0 \mathrm{~K}$, as described in details in Ref. [39, 40].

More details on our approach are reported in Ref. [27.

\section{Results}

\subsection{Osmium}

The calculated lattice parameters of h.c.p. Os are first compared with experimental and previous theoretical results from different sources in Tab. 1. The experimental data have been taken from Ref. [7], where the lattice parameters values have been corrected to $293 \mathrm{~K}$ using thermal expansion data. It can be noted that the PBEsol results are in remarkable good agreement with the experiments. There is also a good agreement with previous theoretical results at $0 \mathrm{~K}$, despite the fact that some of the earlier works were using different methods, with the only exception of the early work by Fast et al. whose LDA values appear to be higher than all other LDA results.

Lattice dynamics results for h.c.p. Os are plotted in Fig. 11 in the LDA approximation. The calculated phonon dispersion is similar to that obtained by Deng et al. [17. The only available experimental point at $\Gamma$ is also shown for comparison and is about $5 \mathrm{~cm}^{-1}$ lower than the calculated LDA value. The phonon dispersion is also similar to that of other h.c.p. metals such as Re and Tc [27] which are close to Os in the periodic table. An important difference is, however, the absence in Os of soft modes at $\Gamma$ and $\mathrm{H}$ contrary to what has been found in both Re and Tc. At these same points, the calculated Grüneisen parameters of Os (Fig. 2) show no anomalous peaks contrary to Re and Tc, hence the phonon frequencies do not rapidly become imaginary when increasing the volume. We note that no negative values of the Grüneisen parameters occur in agreement with positive values of the thermal expansion tensor at any temperature (see below).

The pressure shift of the TO mode measured at $\Gamma$ using Raman spectroscopy and three different pressure-transmitting media (Ref. [3]) are compared to our results in Fig. 3. The frequencies where calculated at $300 \mathrm{~K}$ and for each pressure value using the equilibrium geometry obtained by minimizing the Gibbs energy. It can be noted that the experimental data are in between the LDA and PBE calculated values, with the PBEsol results in better agreement. The experimental values show some scatter, in particular the data set obtained using $\mathrm{KCl}$ as a pressure-transmitting medium are closer to our PBEsol results. The calculated temperature dependence of the TO mode is, however, similar to the experimental results obtained using helium 
Table 1: Comparison between structural parameters of h.c.p. Os from different sources. Experimental data are taken from Ref. [7] and references therein. They have been corrected to $293 \mathrm{~K}$ using thermal expansion data. Previous theoretical results are also reported for comparison (Liu et al. 2011 [18, Ma et al. 2005 [19, Sahu et al. 2005 [41], Deng et al. 2009 [42, Cynn et al. 2002 [20], Fast et al. 1995 [43]). US is for ultrasoft pseudopotentials, PAW for projector-augmented-wave pseudopotentials, FPLMTO for full-potential linear muffin-tin orbitals, FPLAP for full-potential linearized augmented plane-waves.

Method

$\begin{array}{lll}a & c & c / a\end{array}$

PAW, LDA (0 K, this work)

$2.7164 \quad 4.2879 \quad 1.5785$

PAW, PBEsol (0 K, this work)

$2.7284 \quad 4.3062 \quad 1.5783$

PAW, PBE (0 K, this work)

$2.7540 \quad 4.3441 \quad 1.5774$

PAW, LDA (293 K, this work)

$\begin{array}{lll}2.7200 & 4.2966 & 1.5796\end{array}$

PAW, PBEsol (293 K, this work)

$2.7321 \quad 4.3154 \quad 1.5795$

PAW, PBE (293 K, this work)

$2.7579 \quad 4.3540 \quad 1.5787$

US, LDA (0 K, Liu et al. 2011)

$2.7135 \quad 4.2737 \quad 1.575$

US, PBE (0 K, Liu et al. 2011)

$2.7489 \quad 4.3350 \quad 1.577$

US, PBE (0 K, Deng et al. 2009)

$\begin{array}{lll}2.745 & 4.328 & 1.577\end{array}$

US, PBE (0 K, Ma et al. 2005)

$\begin{array}{lll}2.7507 & 4.3388 & 1.5773\end{array}$

FPLAP, LDA (0 K, Sahu et al. 2005) $\quad 2.7204 \quad 4.3112 \quad 1.5847$

FPLAP, PBE (0 K, Sahu et al. 2005) $\quad 2.7576 \quad 4.3667 \quad 1.5835$

FPLMTO, LDA (0 K, Cynn et al. 2002) $2.7165 \quad 4.3029 \quad 1.5840$

FPLMTO, LDA (0 K, Fast et al. 1995) $\quad 2.752 \quad 4.344 \quad 1.578$

Exp. (Owen et al. 1935)

$2.7361 \quad 4.3189 \quad 1.5785$

Exp. (Owen and Roberts 1936)

$\begin{array}{lll}2.7357 & 4.3191 & 1.5788\end{array}$

Exp. (Owen and Roberts 1937)

$\begin{array}{lll}2.7355 & 4.3194 & 1.579\end{array}$

Exp. (Finkel et al. 1971)

$\begin{array}{lll}2.7346 & 4.3174 & 1.5788\end{array}$

Exp. (Rudman 1965)

$\begin{array}{lll}2.7341 & 4.3188 & 1.5796\end{array}$

Exp. (Swanson et al. 1955)

$\begin{array}{lll}2.7342 & 4.3198 & 1.5799\end{array}$

Exp. (Mueller and Heaton 1961)

$\begin{array}{lll}2.7345 & 4.3200 & 1.5798\end{array}$

Exp. (Taylor et al. 1961)

$\begin{array}{lll}2.7342 & 4.3201 & 1.5800\end{array}$

Exp. (Schroeder et al. 1972)

$\begin{array}{lll}2.7340 & 4.3198 & 1.5800\end{array}$

Exp. (Liu et a. 2011)

$\begin{array}{lll}2.7356 & 4.3198 & 1.579\end{array}$ 
and methanol/ethanol, which appear to be consistent with each other. The values of the equilibrium lattice parameters $a$ and $c / a$ at $300 \mathrm{~K}$ as a function of pressure obtained from the minimization of the free energy are reported in Tab. 2. The frequencies in Fig. 3 were computed at these lattice parameters values

Table 2: Structural parameters of h.c.p. Os at $300 \mathrm{~K}$ as a function of pressure according to different DFT functionals. The values reported here correspond to those for which the frequencies at the $\Gamma$ point in Fig. 3 were obtained.

$\begin{array}{ccccccc}\begin{array}{c}\text { Pressure } \\ (\mathrm{GPa})\end{array} & a(\mathrm{LDA}) & c / a(\mathrm{LDA}) & a(\mathrm{PBEsol}) & c / a(\mathrm{PBEsol}) & a(\mathrm{PBE}) & c / a(\mathrm{PBE}) \\ 0 & 2.7201 & 1.5796 & 2.7321 & 1.5796 & 2.7580 & 1.5788 \\ 2 & 2.7158 & 1.58 & 2.7277 & 1.5799 & 2.7531 & 1.5791 \\ 4 & 2.7116 & 1.5803 & 2.7233 & 1.5802 & 2.7483 & 1.5795 \\ 6 & 2.7075 & 1.5806 & 2.7191 & 1.5805 & 2.7436 & 1.5798 \\ 8 & 2.7035 & 1.5809 & 2.7149 & 1.5808 & 2.7391 & 1.5801 \\ 10 & 2.6996 & 1.5812 & 2.7108 & 1.5811 & 2.7346 & 1.5805 \\ 12 & 2.6957 & 1.5815 & 2.7068 & 1.5814 & 2.7303 & 1.5808 \\ 14 & 2.6920 & 1.5818 & 2.7029 & 1.5817 & 2.7261 & 1.5811 \\ 16 & 2.6883 & 1.5821 & 2.6991 & 1.582 & 2.7219 & 1.5814 \\ 18 & 2.6847 & 1.5823 & 2.6954 & 1.5823 & 2.7179 & 1.5816 \\ 20 & 2.6811 & 1.5826 & 2.6917 & 1.5825 & 2.7139 & 1.5819\end{array}$

As outlined in the previous section, we first performed quasi-harmonic calculations assuming a constant $c / a$ ratio. As an example of the results obtained in this way, we show in Fig. 4 the isobaric heat capacity of h.c.p. Os calculated using both the LDA and PBE functionals. The figure also shows available experimental data for comparison. The points from Arblaster [44] are not pure experimental data but the result of a critical evaluation of the available experiments up to $1300 \mathrm{~K}$. It is noteworthy that the electronic contribution, as common for metals, is not negligible and is essential to reach a satisfactory agreement with the experimental data. It can be remarked that the agreement is good at low temperature, while the calculated line departs from the experimental points at high temperatures. This is not unexpected, since the quasiharmonic approximation breaks down when higher-order anharmonic contributions become important at high temperatures. As mentioned in the introduction, theoretical calculations for thermodynamic properties of h.c.p. Os were reported by Liu et al. [18] and Deng et al. [17. The former however did not report any isobaric heat capacity results. The results of Deng et al., are shown in Fig. 4 (olive line) and they are very close to ours without including the electronic contribution. It is noteworthy that they used a different EOS (Birch) and they computed volume-dependent phonon frequencies relaxing the $c / a$ ratio at each fixed volume. They did not compute temperaturedependent lattice parameters and the linear thermal expansions.

Next, we computed anisotropic properties using the full $(a, c / a)$ grid. The temperature dependence of the lattice parameters obtained in this way is reported in Fig. 5. As found for other systems [27, 39], the temperature dependence of the quasi-harmonic results is in good agreement with the experiments, while the discrepancy between the calculated and experimental values can be mostly ascribed to the exchange-correlation functional. In fact, LDA and PBE results essentially "bracket" the experimental values in the temperature range of the figure. The experimental values from Owen et al. [8] 

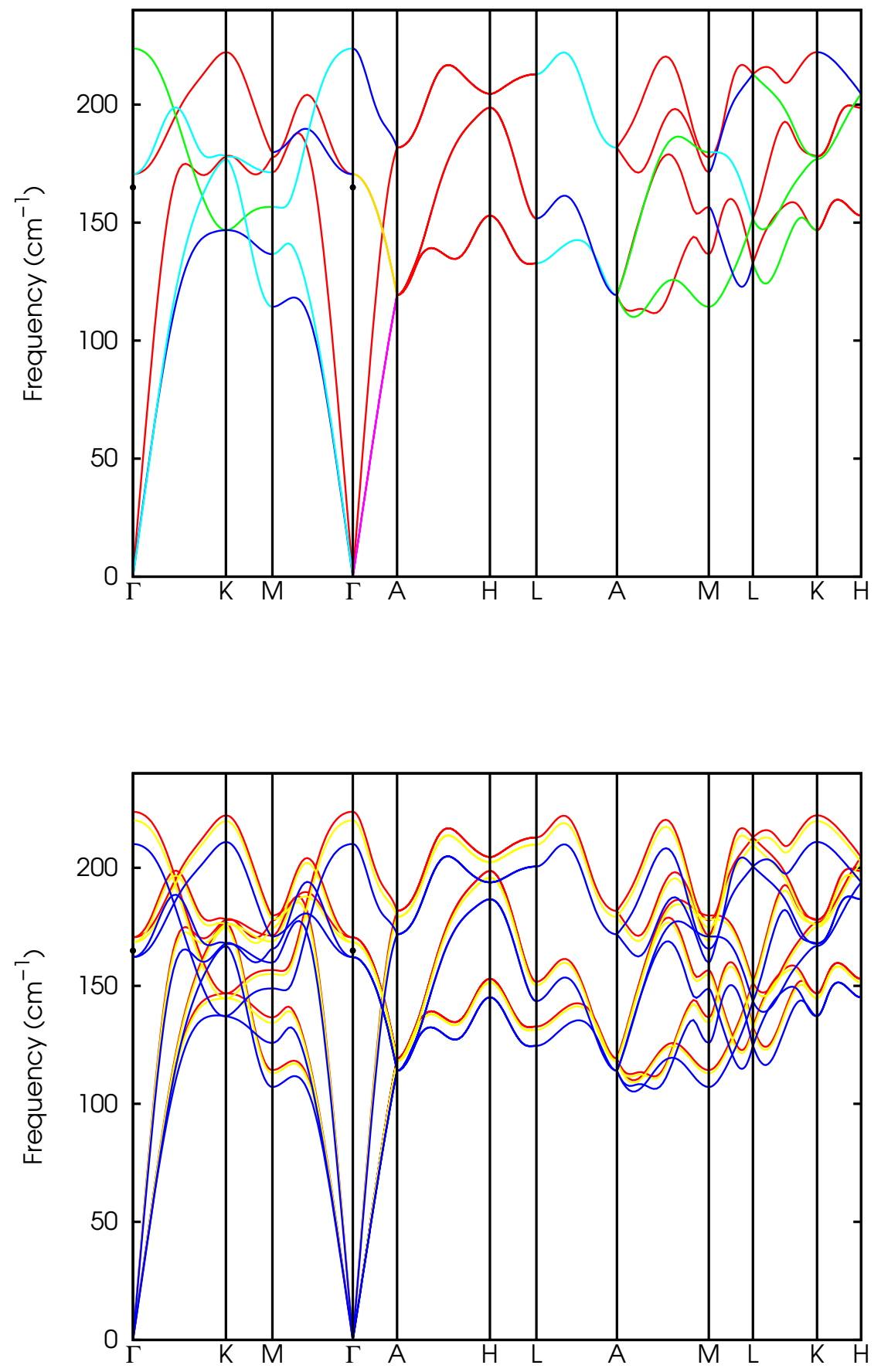

Figure 1: (a) Phonon dispersions of h.c.p. Os calculated with LDA exchangecorrelation functional. The colours represent the different simmetries of the phonon branches (for details see the thermo_pw documentation); (b) Phonon dispersions of h.c.p. Os calculated with LDA (red line), PBEsol (yellow line) and PBE (blue line) exchange-correlation functionals. All calculations were carried out interpolating phonon dispersions at the equilibrium lattice parameters at $300 \mathrm{~K}$. 


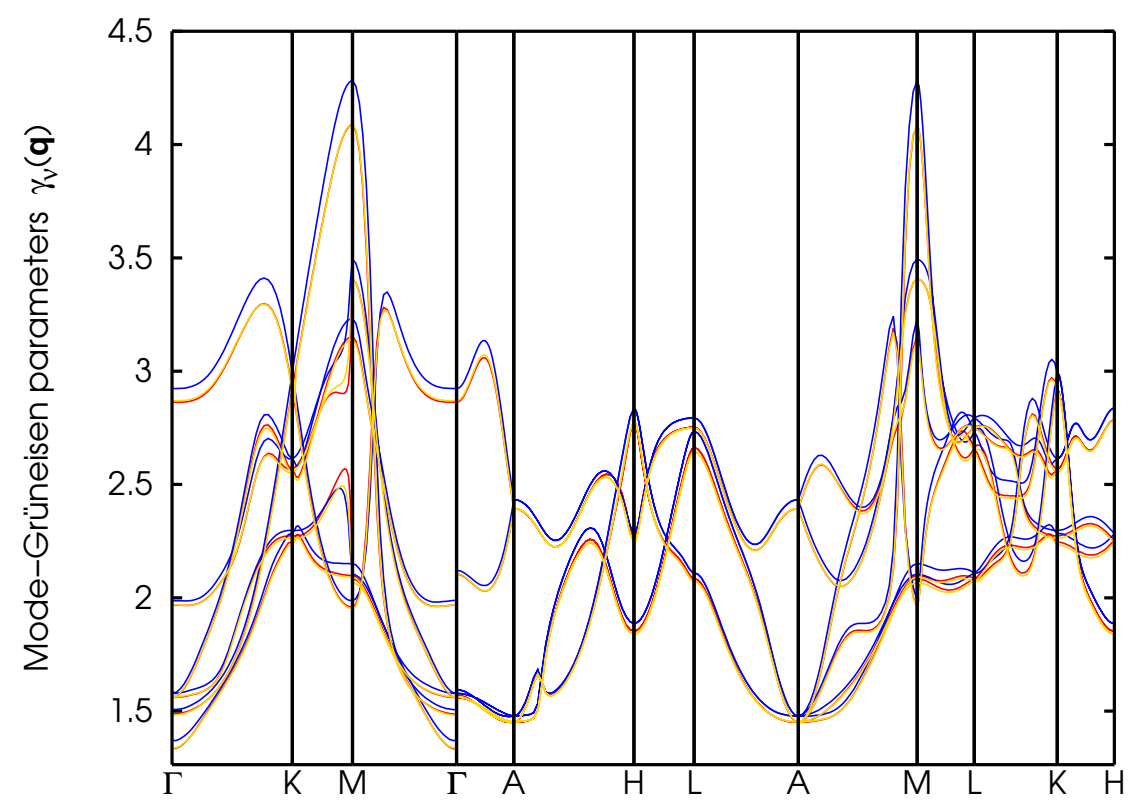

Figure 2: Volume-dependent mode Grüneisein parameters of h.c.p. Os calculated with LDA (red line), PBEsol (yellow line) and PBE (blue line) exchange-correlation functionals at the equilibrium lattice parameters at $300 \mathrm{~K}$.

are lower than other data sets, but within the difference between LDA/PBE calculated results. We remark that the PBEsol results are in remarkable good agreement with the experimental lattice parameters and within the experimental scatter. The behavior of the $c / a$ ratio is similar to other h.c.p. elements [27], i.e. its variation with temperature is very small ( $<1 \%$ from 0 to $2000 \mathrm{~K}$ in the LDA results) and compares satisfactorily with the experiments. No previously calculated result is available.

The results for the thermal expansion tensor are shown in Fig. 6. We report only the "assessed" values from Arblaster [44] for comparison. The values are obtained from a polynomial fit of the original experimental data of lattice parameters (shown in Fig. 5). The calculated results appear to be somehow controversial. The PBE results for $\alpha_{1}$ are in satisfactory agreement with Arblaster's assessed values, while LDA values underestimate them. On the contrary, both LDA and PBE calculated results for $\alpha_{3}$ do not reproduce well Arblaster's assessed values.

\subsection{Ruthenium}

The calculated lattice parameters of h.c.p. $\mathrm{Ru}$ are first compared with experimental the theoretical results from different sources in Tab. 3. The experimental data have been taken from Ref. [48], where the lattice parameters values have been corrected to $293 \mathrm{~K}$ using thermal expansion data. The PBEsol results are in better agreement with the experiments than the LDA and PBE values. Contrary to Os, however, the PBEsol values are slightly lower than the experimental values. Theoretical results at $0 \mathrm{~K}$ from Heid et al. [5] using an LDA functional and a norm-conserving Hamann-Schüter-Chiang 


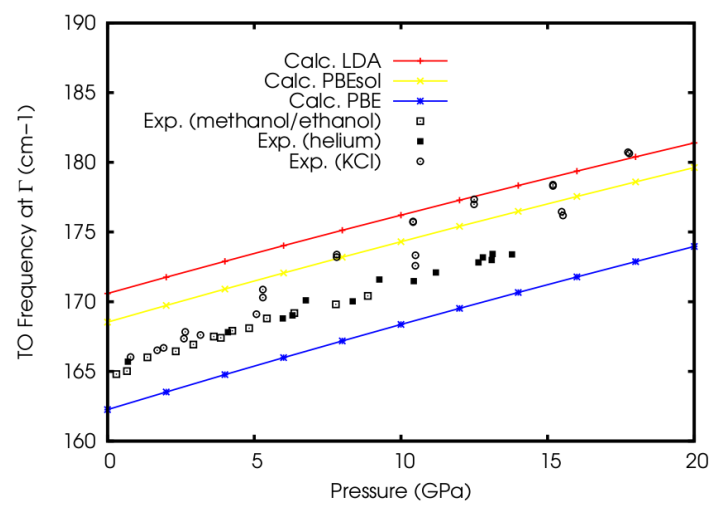

Figure 3: Pressure shift of the TO phonon frequency at the $\Gamma$ point and $\mathrm{T}=300 \mathrm{~K}$ with LDA, PBEsol and PBE functionals. Experimental data were obtained from Raman spectroscopy in Ref. [3] and using three different pressure-transmitting media as in the legend.
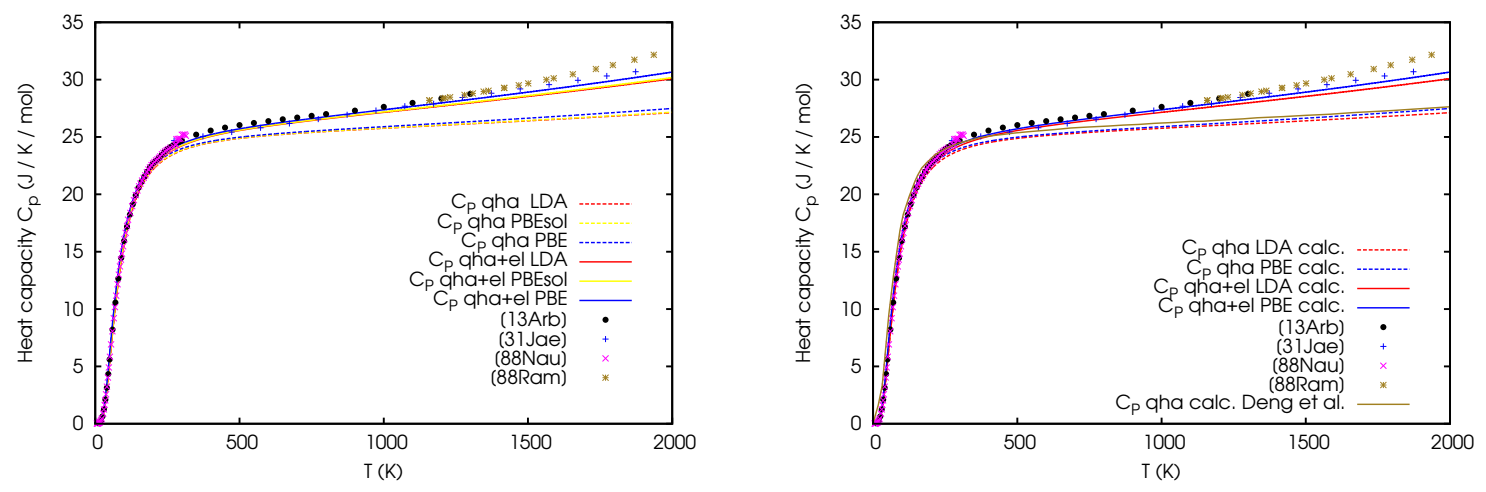

Figure 4: (a) Calculated isobaric heat capacity of h.c.p. Os as a function of temperature with LDA/PBEsol/PBE exchange-correlation functionals, quasi-harmonic only $\left(C_{P}\right.$ qha) and quasi-harmonic plus electronic $\left(C_{P}\right.$ qha $\left.+\mathrm{el}\right)$. The calculations were done assuming a constant $c / a$ ratio and using the Murnaghan EOS. Experimental data from different sources are shown as points: 31Jae [45], 88Nau [46] and 88Ram [47. Points from 91Arb (ref. [44]) are not pure experimental data but assessed values obtained from a critical evaluation of available experimental data; (b) As in (a) but including previously calculated data from Deng et al. (and removing PBEsol results for clarity). 

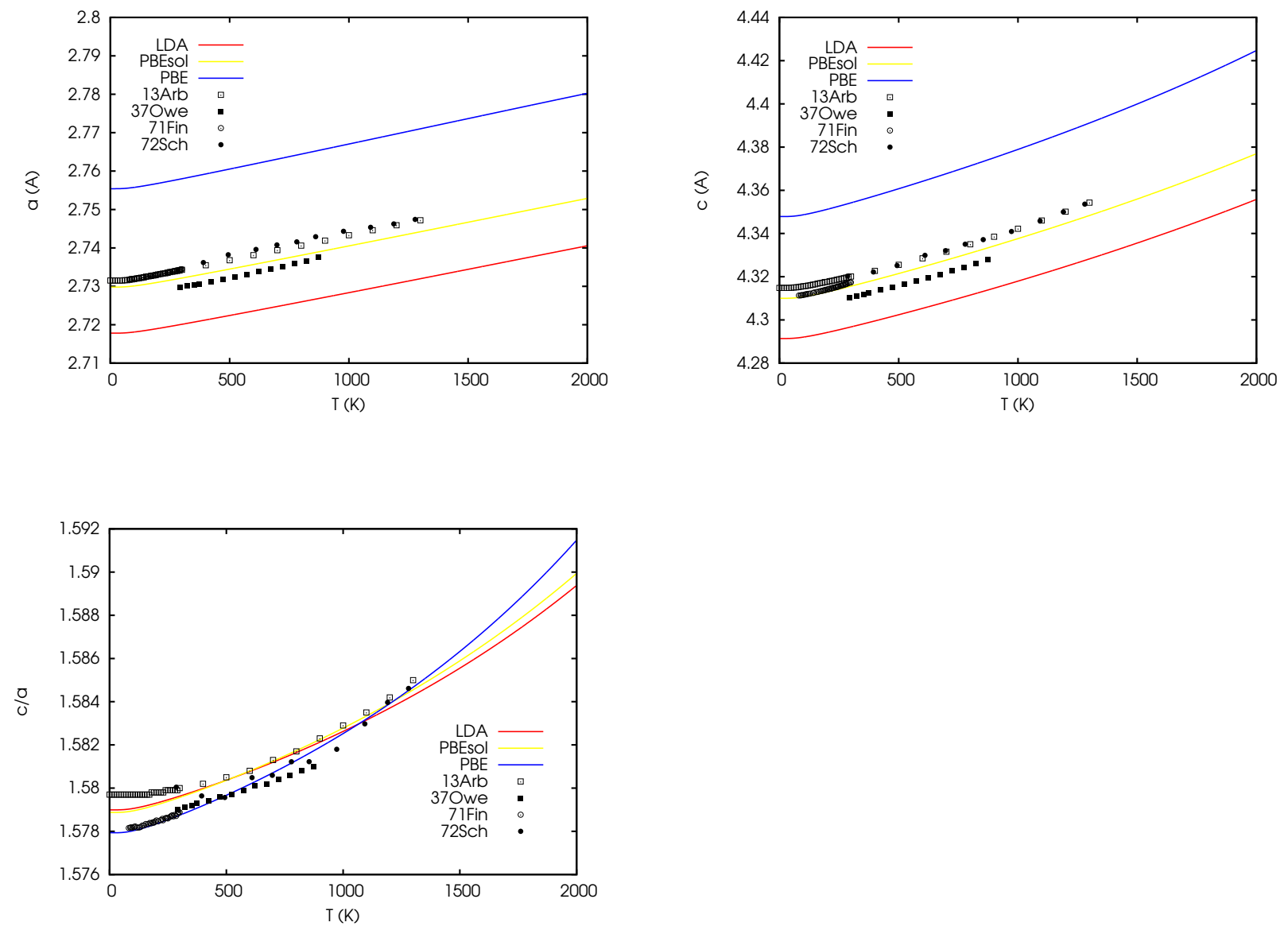

Figure 5: Quasi-harmonic variation with temperature of the lattice parameters $a, c$ and $c / a$ of h.c.p. Os with both LDA and PBE functionals. These results were obtained using the full grid $(a, c / a)$ and minimizing the Helmholtz energy to obtain $a(T), c(T)$ and $c / a(T)$. Experimental data from different sources are shown as points: 37Owe [8], 71Fin [9], 72Sch [10]. Points from 13Arb [7] are not pure experimental data but assessed values obtained from a critical evaluation of available experimental data. 

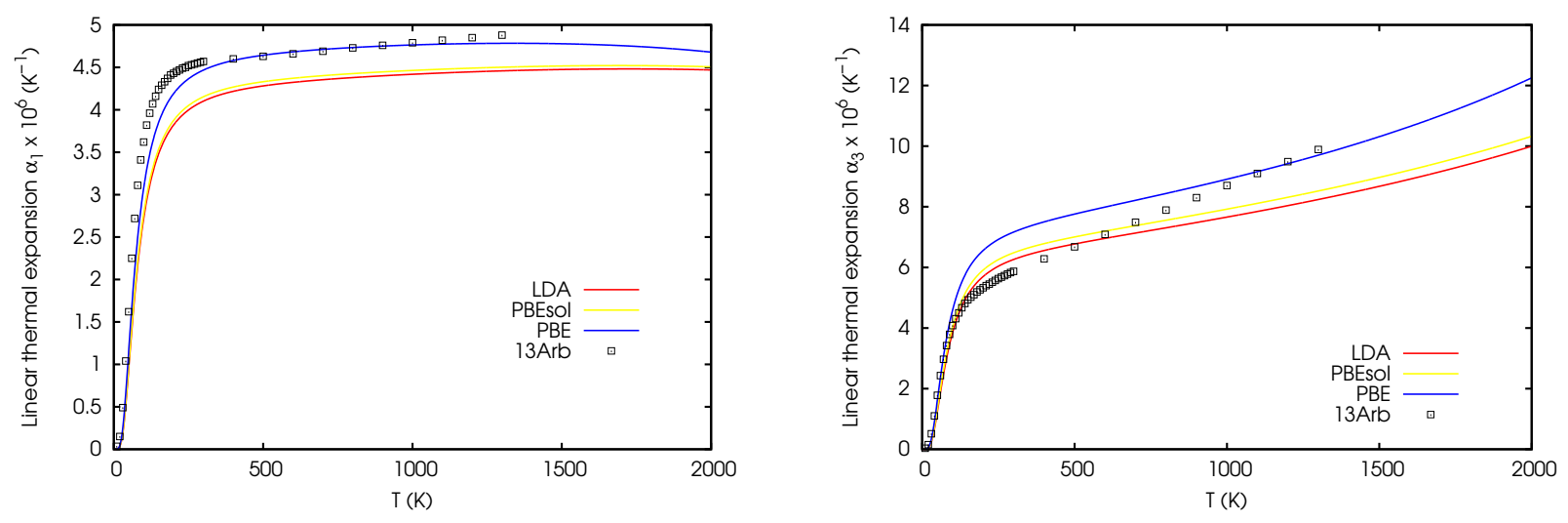

Figure 6: Calculated linear thermal tensor $\left(\alpha_{1}=\alpha_{2}\right.$ and $\left.\alpha_{3}\right)$ for Os h.c.p. These results were obtained using the full grid $(a, c / a)$, minimizing the Helmholtz energy to obtain $a(T)$ and $c / a(T)$. Points from 13Arb (ref. [7]) are not pure experimental data but assessed values obtained from a critical evaluation of available experimental data.

pseudopotential appear to be significantly lower than our values, possibly because of the different pseudopotential constructed using a mixed-basis set. The results from Yaozhuang et al., also using a norm-conserving pseudopotential and an LDA functional, appear to be closer to ours for the lattice parameter $a$, but significantly differ for $c$. Their results for the latter parameter (and for the $c / a$ ratio) are also quite different from all experimental results. Finally, the results from Souvatzis et al. were obtained using a PAW pseudopotential and PBE functional. As can be seen from Tab. 3, they overestimate both our results (PBEsol and PBE) and all experimental results.

Contrary to Os, experimental values of the phonon frequencies are available for h.c.p. $\mathrm{Ru}$ and are shown in Fig. 7 together with the theoretical results calculated using LDA, PBEsol and PBE functionals. The LDA frequencies overestimate the experimental values in most branches, up to nearly $20 \mathrm{~cm}^{-1}$. The PBE results are in better agreement with the experimental frequencies, although a slight difference is still present at the high frequency optical branches where they underestimate the experiments. PBEsol frequencies agree fairly well with the experiments for almost every branch. This functional dependence of the phonon frequencies is similar to what was found in several cubic metals [49]. The phonon dispersions in Fig. 7 also agree well with the results calculated by Heid et al. [5] using density-functional-perturbation theory and by Souvatzis et al. [23] using a $4 \times 4 \times 3$ supercell. As an example, we report in Fig. 8 a comparison of previously calculated frequencies and experimental data along the $\Gamma-\mathrm{M}$ direction in the $\mathrm{BZ}$, where some anomalies with respect to other h.c.p. elements have been found in the vicinity of the M point: (1) the longitudinal modes (Fig. 3.2 show a sudden drop in frequency when approaching the M point; (2) the acoustic branch of the transversal modes $\Sigma_{4}$ (Fig. 3.2) are essentially flat for about $1 / 3$ of the $\Gamma$-M distance; (3) the z-polarized branches $\Sigma_{3}$ are nearly degenerate at the $\mathrm{M}$ point. All points are well reproduced by all calculated results. The agreement with the experimental data is also remarkably good for all calculated frequencies, with Souvatzis' calculated frequencies which appear slightly worse than Heidi's and ours, 
Table 3: Comparison between structural parameters of h.c.p. Ru from different sources. Experimental data are taken from Ref. [48] and references therein. They have been corrected to $293 \mathrm{~K}$ using thermal expansion data. Previous theoretical results are also reported for comparison.

Method

$$
a
$$$$
c \quad c / a
$$

PAW, LDA (0 K, this work) $\quad 2.6770 \quad 4.2260 \quad 1.5786$

PAW, PBEsol (0 K, this work) $\quad 2.6920 \quad 4.2485 \quad 1.5784$

PAW, PBE (0 K, this work) $\quad 2.7219 \quad 4.2934 \quad 1.5774$

PAW, LDA (293 K, this work) $\quad 2.6820 \quad 4.2363 \quad 1.5795$

PAW, PBEsol (293 K, this work) $2.6967 \quad 4.2598 \quad 1.5796$

PAW, PBE (293 K, this work) $\quad 2.7273 \quad 4.3056 \quad 1.5787$

NC, LDA (0 K, Heid et al.) $\quad 2.701 \quad 4.270 \quad 1.581$

PAW, GGA (0 K, Souvatzis et al.) $\quad 2.74 \quad 4.32 \quad 1.577$

NC, LDA (0 K, Yaozhuang et al.) $\quad 2.691 \quad 4.322 \quad 1.606$

Exp. (Owen et al. 1935) $\quad 2.7044 \quad 4.2818 \quad 1.5833$

Exp. (Owen and Roberts 1936) $\quad 2.7042 \quad 4.2819 \quad 1.5834$

Exp. (Owen and Roberts 1937) $\quad 2.7040 \quad 4.2819 \quad 1.5835$

Exp. (Ross et al.) $\quad 2.7042 \quad 4.2799 \quad 1.5827$

Exp. (Finkel et al. 1971) $\quad 2.7062 \quad 4.2815 \quad 1.5821$

Exp. (Hellawell et al. 1954) $\quad 2.7058 \quad 4.2817 \quad 1.5824$

Exp. (Swanson et al. 1955) $\quad 2.7059 \quad 4.2819 \quad 1.5824$

Exp. (Hall et al. 1957) $\quad 2.7058 \quad 4.2805 \quad 1.5820$

Exp. (Anderson et al. 1960) $\quad 2.7058 \quad 4.2814 \quad 1.5823$

Exp. (Cernohorsky 1960) $\quad 2.7059 \quad 4.2812 \quad 1.5822$

Exp. (Savitskii et al. 1962) $\quad 2.7059 \quad 4.2819 \quad 1.5824$

Exp. (Schroeder et al. 1972) $\quad 2.7056 \quad 4.2826 \quad 1.5829$ 
despite the fact that different functionals were used and some values were obtained at $0 \mathrm{~K}$. The fact can possibly be explained noting that Heidi's calculated LDA lattice parameters at $0 \mathrm{~K}$ are very close to our PBEsol calculated values at $298 \mathrm{~K}$.

The Grüneisen parameters calculated using the LDA, PBEsol and PBE functionals are shown in Fig. 9. They are remarkably similar to those of Os, with the highest values at the $\mathrm{M}$ point and ranging from slightly less than 1.5 to approximately 3.5 .
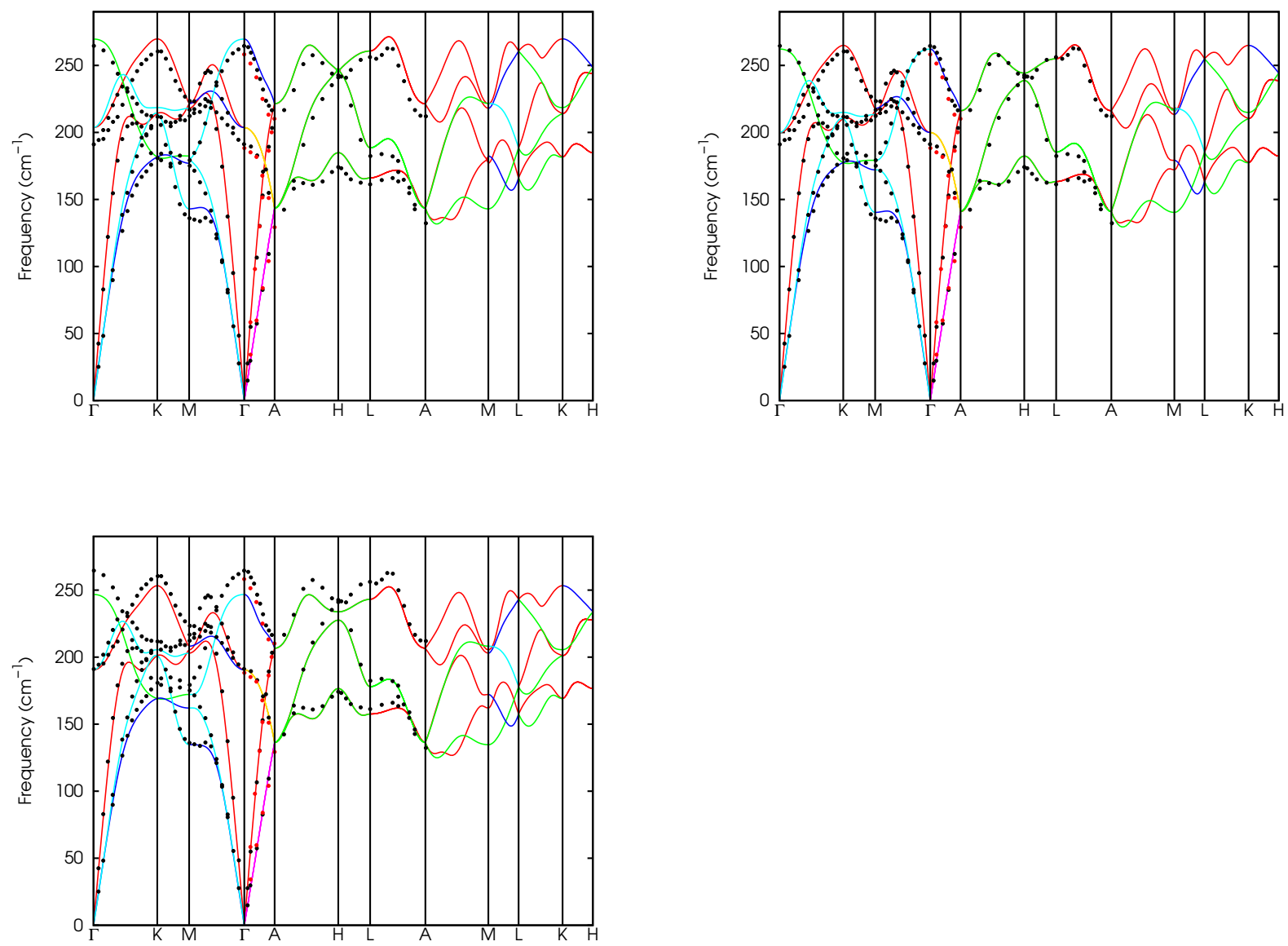

Figure 7: Phonon dispersion curves of Ru along high symmetry points and directions in the BZ calculated with LDA (a), PBEsol (b) and PBE (c) exchange-correlation functionals using a mesh of $6 \times 6 \times 4$ q-points. The colours represent the different simmetries of the phonon branches (for details see the thermo pw documentation). The calculation was carried out at the equilibrium lattice parameters at $298 \mathrm{~K}(a=2.6820$ $\AA$ and $c / a=1.5795$ for LDA, $a=2.6967 \AA$ and $c / a=1.5796$ for PBEsol, $a=2.7273$ $\AA$ and $c / a=1.5787$ for PBE). Experimental data are from Ref. [5] (black circles) and Ref. [6] (red circles).

As for Os, we first carried out calculations of isotropic quantities of h.c.p. Ru using Murnaghan EOS and a constant $c / a$ ratio. The results for the isobaric heat capacity, for which several experimental data are available, are shown in Fig. 3.2. It must first be remarked that the experimental values from Jaeger et al. 45] are significantly lower compared to all other data in the temperature range 500-1500 K. Furthermore, the low temperature data from Clusius [50] show a trend at high temperatures which 

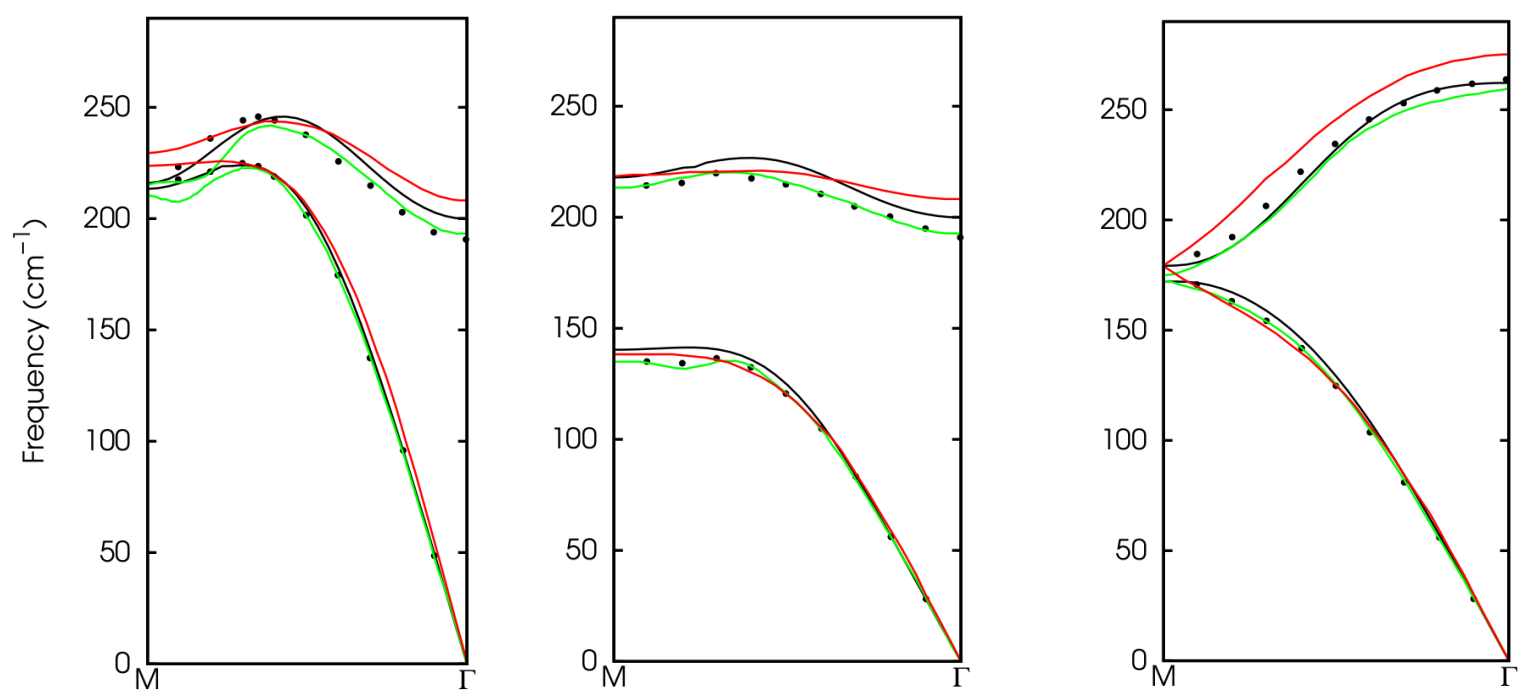

Figure 8: Phonon dispersion curves of $\mathrm{Ru}$ along the $\mathrm{\Gamma}-\mathrm{M}$ direction in the $\mathrm{BZ}$ calculated as in this work at $298 \mathrm{~K}$ using DFPT and the PBEsol functional (black lines), as in Heid et al. [5] at $0 \mathrm{~K}$ using DFPT and an LDA functional (yellow lines) and as in Souvatzis et al. 23] at $0 \mathrm{~K}$ using a the supercell method and a GGA functional (red lines). Experimental data are from Ref. [5] (black circles). (a) longitudinal modes $\Sigma_{1}$, (b) transversal modes $\Sigma_{4}$, (c) transversal modes $\Sigma_{3}$.

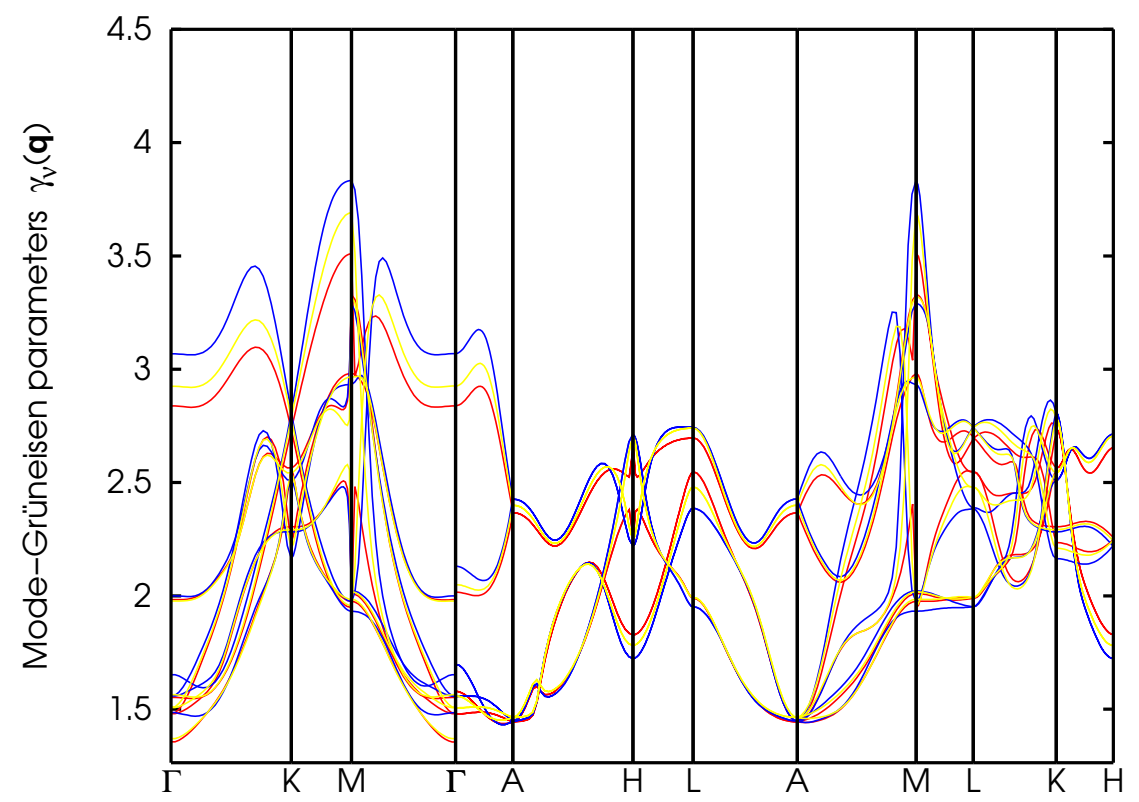

Figure 9: Volume-dependent mode Grüneisein parameters of h.c.p. Ru calculated with LDA (red line), PBEsol (yellow line) and PBE (blue line) exchange-correlations functional at the equilibrium lattice parameters at $300 \mathrm{~K}$. 
conflicts with other experimental values above $300 \mathrm{~K}$. In fact, Arblaster discussed this issue in his work and his "assessed" values (also reported in the figure) have been chosen to properly link low temperature data from Clusius [50] to high temperature data. It is remarkable that our first-principles PBE results are nearly identical to Arblaster's values up to $500 \mathrm{~K}$, thus confirming his choice of recommended values. Above this temperature, both LDA and PBE calculated results start deviating from the experimental points and at high temperature the calculations largely underestimate the experiments. It is noteworthy that the electronic contribution to the heat capacity of $\mathrm{Ru}$ is not negligible and necessary, though not sufficient, to improve the agreement with the experimental data. It is also worth noting that there is a significant difference in this contribution for h.c.p. Ru between the LDA and PBE results. This arises from the shift of the electronic DOS computed at different (minimum) lattice parameters when the calculation is done with different functionals. Fig. 3.2 shows an enlargement of the isobaric heat capacity results in the temperature range 0-1000 K, including previously calculated results by Yaozhuang et al. (for the sake of clarity, our results including the electronic contribution have been removed). It is clear that our heat capacity values agree well with those computed by Yaozhuang et al. at low temperatures, whereas above approximately room temperature the latters are higher and closer to the experimental data without including the electronic contribution. These authors state in their paper that they computed the heat capacity for the anisotropic h.c.p. Ru using Eq. 6 as in our present work. In this equation, they used the volumetric thermal expansion obtained from anisotropic linear thermal expansions as $\beta=2 \alpha_{1}+\alpha_{3}$. As we prove in the following (Fig. 14), the difference with the volumetric thermal expansion obtained in our calculations is negligible. Negligible differences are expected also in the values of $C_{V}$, which at room temperature have almost reached the plateau value of $3 R$, with $R$ the gas constant. Yaozhuang et al. used in Eq. 6 the anisotropic bulk modulus obtained from the full elastic constants tensor. They report a temperature-independent value of the bulk modulus, derived from elastic constants computed only at $0 \mathrm{~K}$, equal to $322 \mathrm{GPa}$ at $0 \mathrm{~K}$ and which compares reasonably well with our results (361 and 307 GPa for LDA and PBE respectively using the Murnaghan EOS and including ZPE). In our results the bulk modulus decreases with temperature down to $325 \mathrm{GPa}$ and 271 GPa for LDA and PBE respectively at $1000 \mathrm{~K}$. Hence this may explain the discrepancy between Yaozhuang's and our results for the high temperature isobaric heat capacity, with an additional contribution from differences in the equilibrium volumes, stemming from discrepancies in the calculated lattice parameters (as in Tab. 3). In fact the volume obtained from the $0 \mathrm{~K}$ parameters from Yaozhuang et al. is $27.1^{3}$ and ours, including the ZPE, are 26.3 (LDA) and 27.6 (PBE) ${ }^{3}$. Thus the excellent agreement with the experimental data obtained in Yaozhuang's quasi-harmonic results without electronic contribution is likely fortuitous.

The lattice parameters of h.c.p. $\mathrm{Ru}$ as a function of temperature calculated using the full $(a, c / a)$ grid are reported in Fig. 11. As for Os, the LDA and PBE results "bracket" the experimental values starting from $0 \mathrm{~K}$, with a difference which is due to the exchange-correlation functional. In fact, the PBEsol results are closer to the experiments, but contrary to Os they are still slightly underestimating all data sets. The temperature dependence of the experimental lattice parameters is well reproduced by the quasi-harmonic results up to approximately $1500 \mathrm{~K}$. At higher temperatures the quasi-harmonic approximation breaks down and the experimental values increase more than our results. The variation of the $c / a$ ratio for $\mathrm{Ru}$ is slightly higher than 

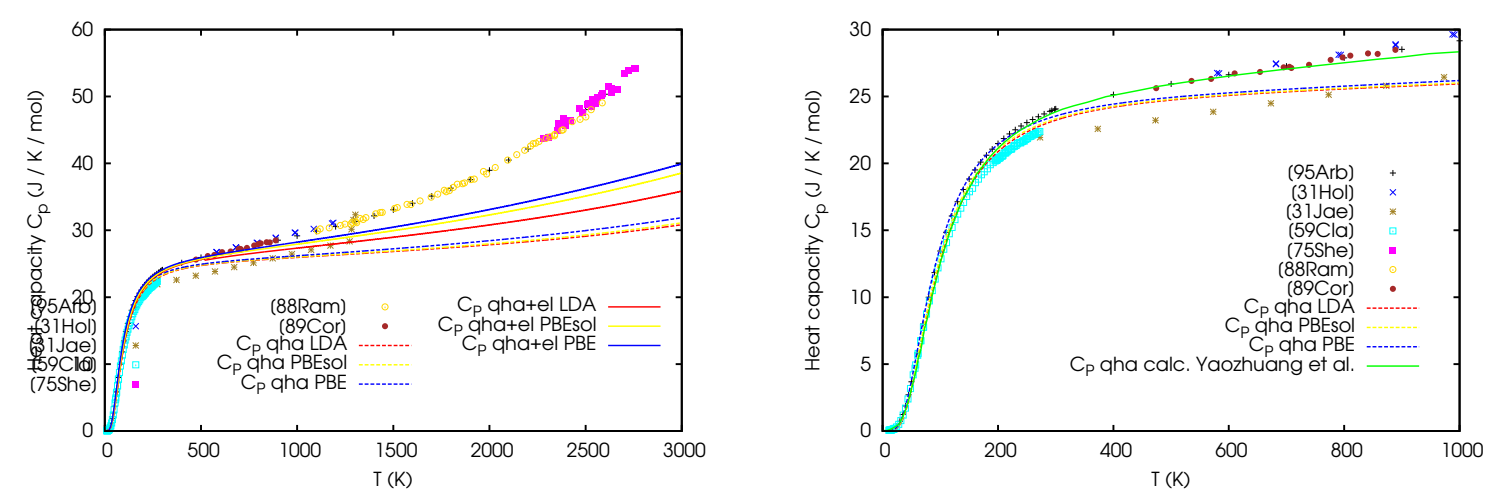

Figure 10: (a) Calculated isobaric heat capacity of h.c.p. Ru as a function of temperature with $\mathrm{LDA} / \mathrm{PBEsol} / \mathrm{PBE}$ exchange-correlation functionals, quasi-harmonic only $\left(C_{P}\right.$ qha) and quasi-harmonic plus electronic $\left(C_{P}\right.$ qha $\left.+\mathrm{el}\right)$. The calculations were done assuming a constant $c / a$ ratio and using the Murnaghan EOS. Experimental data from different sources are shown as points: 31Hol [51], 31Jae [45], 59Clu [50], 75She [52], 88Ram [47] and 89Cor [53]. Points from Arblaster (Ref. [11]) are not pure experimental data but assessed values obtained from a critical evaluation of available experimental data; (b) as in (a) but including previous theoretical results from Yaozhuang et al. (and removing qha + el results for clarity).

for Os $(\sim 1 \%)$ but still very small. The calculated results do not reproduce well the experimental values, though the differences are of the same order of magnitude as those among different experimental data sets. The different temperature dependence of the $c / a$ ratio is probably within the numerical accuracy which can be expected from these calculations.

The anisotropic thermal expansions for h.c.p. $\mathrm{Ru}$ are shown in Fig. 12, At low temperature, a good agreement is obtained between the experimental values and the PBE results, whereas the LDA calculated values are lower in the whole temperature range up to $2000 \mathrm{~K}$. At high temperatures, the calculated thermal expansion along the $a$ direction $\left(\alpha_{1}\right)$ strongly underestimate the experimental values, while the good agreement between the PBE results and the experiments for $\alpha_{3}$ is probably fortuitous. In fact, results for $\alpha_{1}$, the lattice parameters and the heat capacity all suggest that the quasi-harmonic approximation breaks down for $\mathrm{Ru}$ above an approximate temperature which depends on the evaluated quantity and goes from $500 \mathrm{~K}$ for the heat capacity (Fig. 10) to $1000 \mathrm{~K}$ for the lattice parameters (Fig. 11). Our present values of $\alpha_{1}$ and $\alpha_{3}$ agree almost perfectly with the previously calculated values by Yaozhuang et al. 23], while they differ significantly from those by Souvatzis et al., which are also limited to temperatures below $300 \mathrm{~K}$.

We finally point out that the calculation of isotropic quantities, such as volume thermal expansion, is nearly identical when using the Murnaghan EOS and a constant $c / a$ ratio and when using the full $(a, c / a)$ grid. This is shown in Figs. 13, 14. As we already discussed, the variation of the $c / a$ ratio with temperature is limited for both Os and $\mathrm{Ru}$ and hence has limited influence on isotropic quantities. Similar results were also obtained for Re and Tc [27. The thermal expansions for both Os and $\mathrm{Ru}$ are positive in the entire temperature range, in agreement with the calculated 

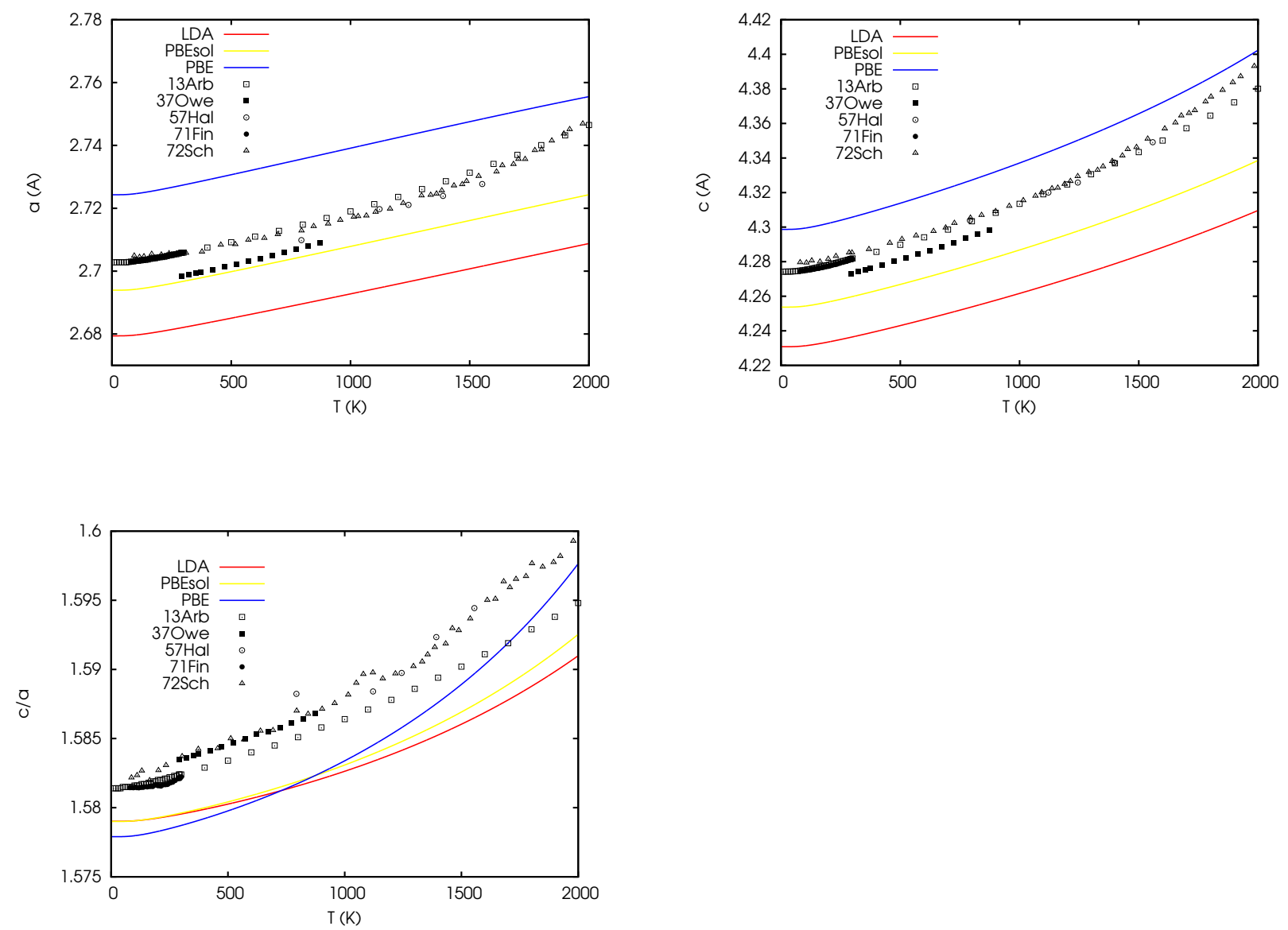

Figure 11: Quasi-harmonic variation of the lattice parameters $a, c$ and $c / a$ of h.c.p. Ru. Experimental data from different sources are shown as points: $37 \mathrm{Owe}$ [8], 57Hal [13], 71Fin [9], 72Sch [10]. Points from 13Arb [48] are not pure experimental data but assessed values obtained from a critical evaluation of available experimental data. These results were obtained using the full grid $(a, c / a)$, minimizing the Helmholtz energy to obtain $a(T), c(T)$ and $c / a(T)$ 

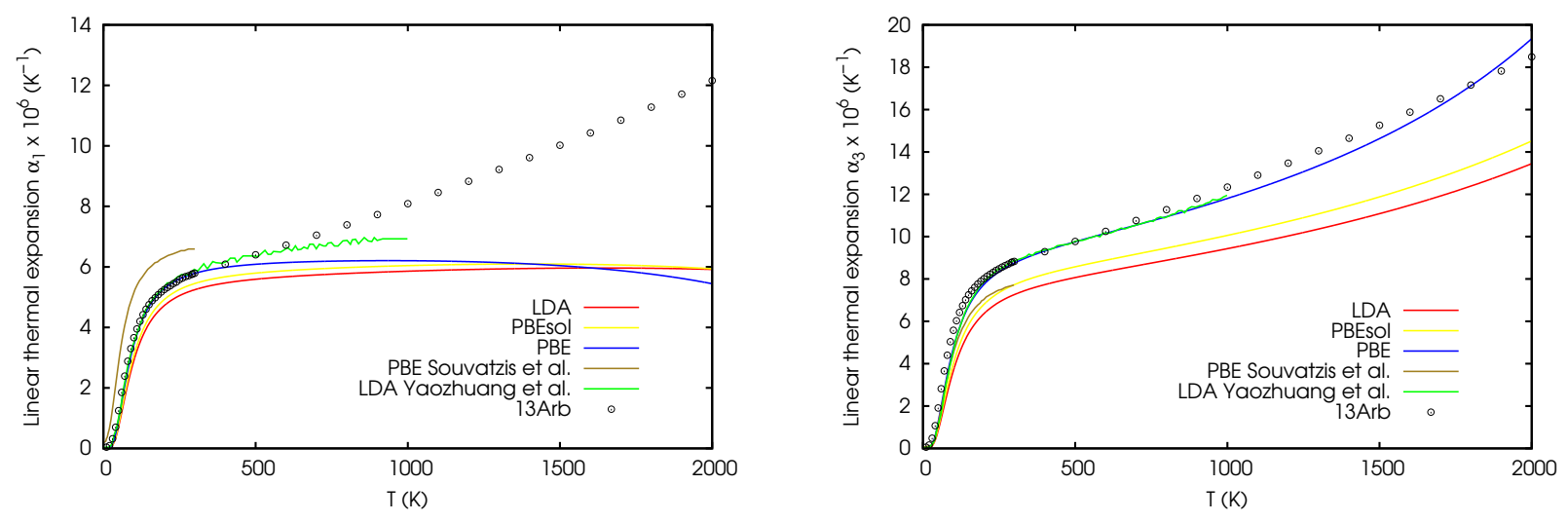

Figure 12: Calculated linear thermal expansion tensor $\left(\alpha_{1}=\alpha_{2}\right.$ and $\left.\alpha_{3}\right)$ for Ru h.c.p. using LDA, PBE and PBEsol functionals. These results were obtained using the full grid $(a, c / a)$, minimizing the Helmholtz energy to obtain $a(T)$ and $c / a(T)$. Points from 13Arb [48] are not pure experimental data but assessed values obtained from a critical evaluation of available experimental data. Previously calculated results from Souvatzis et al. 23] (olive line) and Yaozhuang et al. 24] (green line) are also reported for comparison.

mode Grüneisen parameters. The calculated thermal expansion of Os is also in good agreement with the theoretical results by Deng et al.[17, as it can be seen in Fig. 13. On the contrary, the quasi-harmonic Debye model used in the work by Liu et al. appears to significantly underestimate the volume thermal expansion compared to both full quasi-harmonic results and Arblaster's assessed values.

At the end of our discussion of several results for different quantities, we note that the quasi-harmonic approximation is expected to break down at high temperatures. This is well reported in several papers (and references therein) [39, 54, 36. It is customary to set the Debye temperature $\left(\theta_{D}\right)$ as the breaking point, above which anharmonic contributions beyond the quasi-harmonic approximation start playing a non-negligible role, more recent works suggest that the breaking point is rather system dependent and there are cases in which quasi-harmonic results provide satisfactory values well above $\theta_{D}$. Methods to include additional anharmonic contributions are mostly based on different flavors of molecular dynamics or on including third- or higher-order terms in the perturbation expansion of the internal energy in atomic displacements [55, 56, 39]. These methods are more computational demanding, unless they include some simplifying assumptions. The quasi harmonic approximation still provide reliable results at low/medium temperatures and often represents a starting point for the application of more sophisticated methodologies. A final note on the high-pressure anomalies recently found in h.c.p. Os by Dubrovinsky et al. [16]. As well discussed in their work, these anomalies would require a higher level of theory (DMFT, spin-orbit coupling). The present quasi-harmonic calculations however use phonons calculated at volumes corresponding to the maximum pressure of about $90 \mathrm{GPa}$. Up to this point, the difference between the DMFT results and DFT calculations appear to be negligible. 


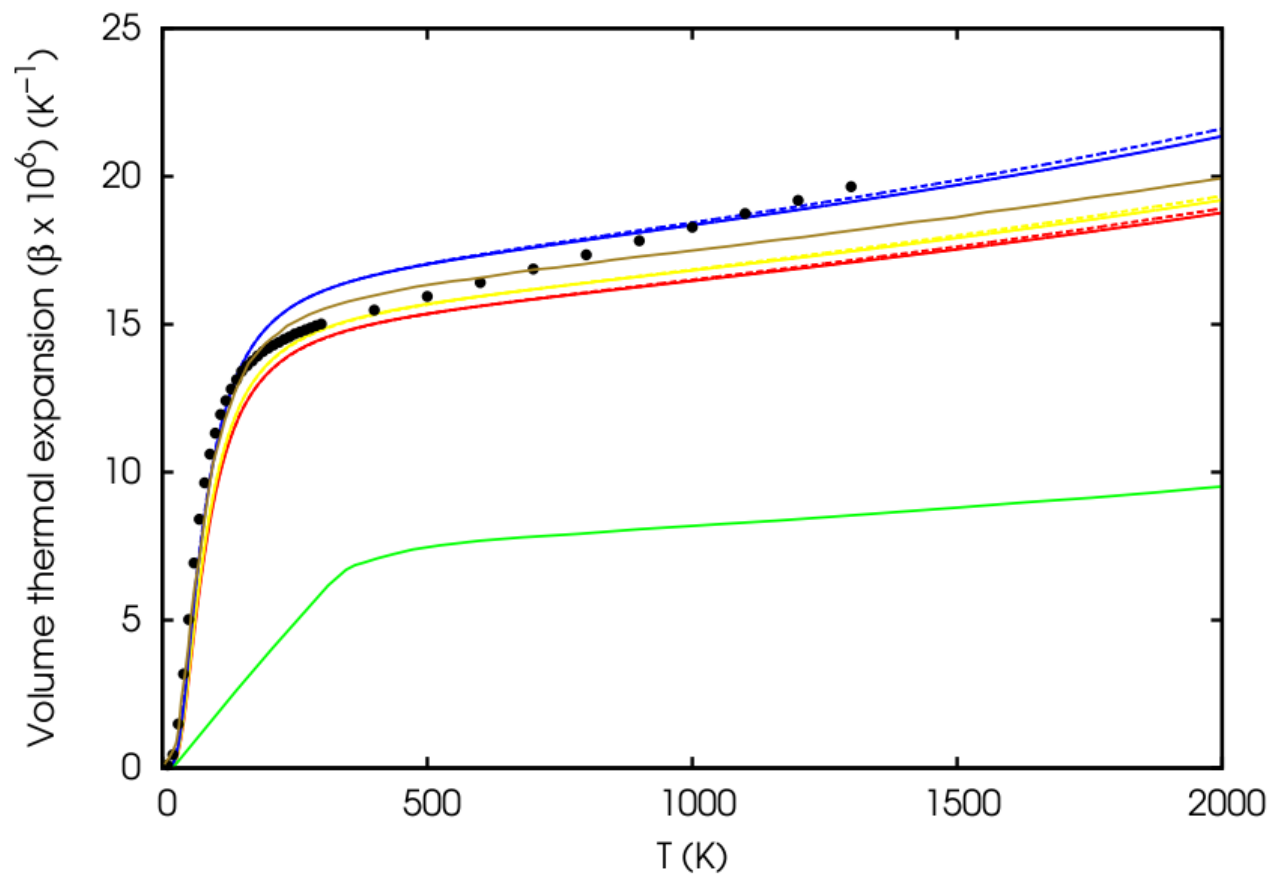

Figure 13: Quasi-harmonic volume thermal expansion of h.c.p. Os calculated as a function of temperature with LDA (red lines), PBEsol (yellow lines) and PBE (blue lines) exchange-correlation functionals. The continuous lines are obtained assuming a constant $c / a$ ratio and using the Murnaghan EOS. The dashed lines are obtained using the full anisotropic results for the linear thermal expansions $\left(\alpha_{1}\right.$ and $\left.\alpha_{3}\right)$ and $\beta=2 \alpha_{1}+\alpha_{3}$. Theoretical results from Deng et al. [17] (olive line) and Liu et al. [18] (green line) are also reported for comparison. 


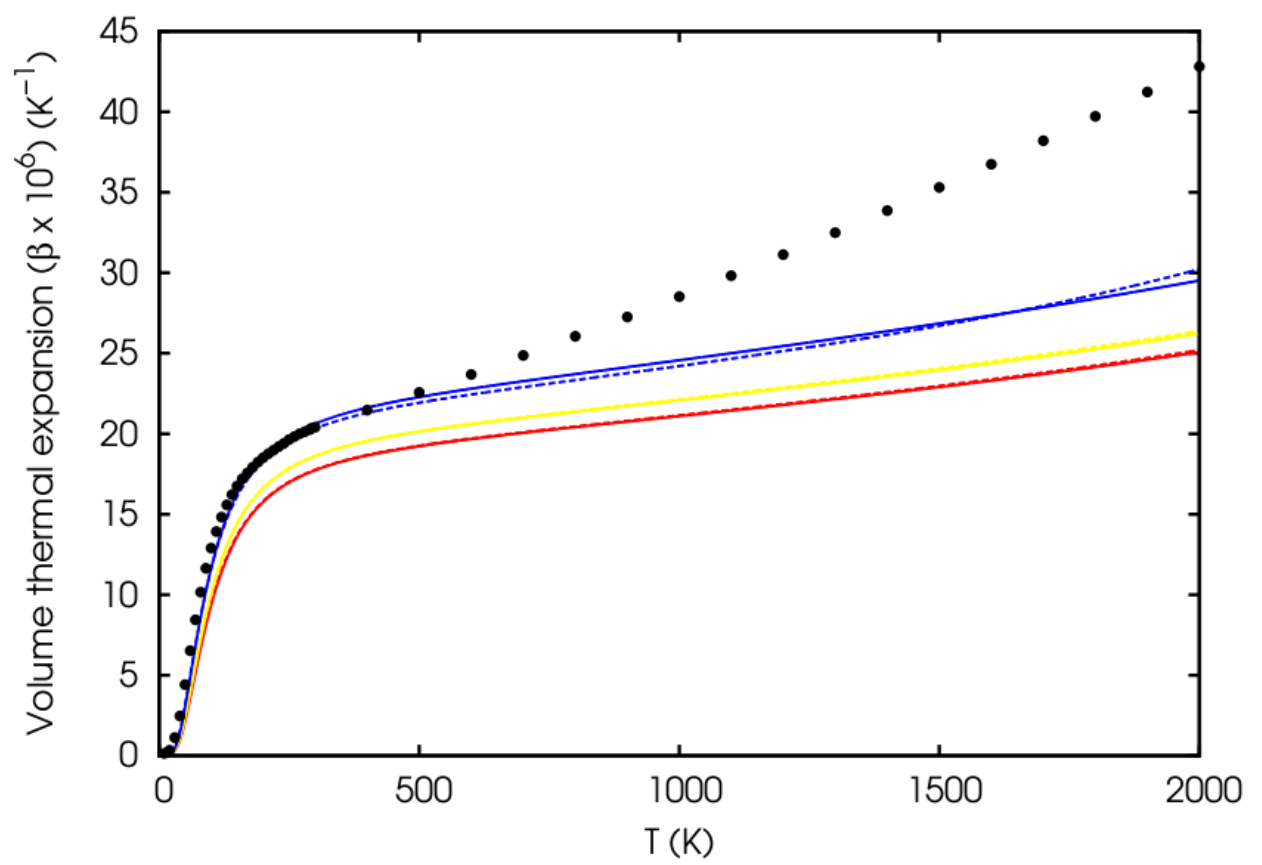

Figure 14: Quasi-harmonic volume thermal expansion of h.c.p. Ru calculated as a function of temperature with LDA (red lines), PBEsol (yellow line) and PBE (blue lines) exchange-correlation functionals. The continuous lines are obtained assuming a constant $c / a$ ratio and using the Murnaghan EOS. The dashed lines are obtained using the full anisotropic results for the linear thermal expansions $\left(\alpha_{1}\right.$ and $\left.\alpha_{3}\right)$ and $\beta=2 \alpha_{1}+\alpha_{3}$. Theoretical results from Yaozhuang et al. 24] (olive line) are also reported for comparison. 


\section{Conclusions}

Using density functional theory and the quasi-harmonic approximation, we analyzed the lattice dynamics and thermophysical properties of h.c.p. Os and Ru. At first, we employed a temperature-independent $c / a$ and the Murnaghan EOS to derive temperaturedependent properties. Afterwards, we carried out quasi-harmonic calculations on a full $(a, c / a)$ grid and obtained fully anisotropic results as the temperature dependence of the lattice parameters and thermal expansion tensor.

Both Os and Ru phonon dispersions do not show soft modes as we obtained for Re and Tc. A good agreement with the experimental phonon frequencies was obtained for h.c.p. Ru. A slight disagreement with experiments remains for $\mathrm{Ru}$ for the optical highfrequency modes. A satisfactory agreement with the experiments was also obtained for the TO pressure shift at the $\Gamma$ point for Os, particularly when using the PBEsol functional.

For Os, the calculated thermophysical quantities show a remarkable agreement with experimental values up to the maximum temperature of $1300 \mathrm{~K}$ where they are available. The lattice parameters as a function of temperature show a difference with experimental values at any temperature which mostly depends on the exchangecorrelation functional, with LDA (PBE) calculations underestimating (overestimating) the experiments, whereas the PBEsol values are essentially on top of experiments. The PBE thermal expansion along the $a$ direction is in satisfactory agreement with the assessed values from Arblaster, while along the $c$ direction no functional is fully in agreement with the recommended values.

For $\mathrm{Ru}$, experimental data of thermodynamic quantities are available up to nearly $3000 \mathrm{~K}$ and the quasi-harmonic approximation appears to break down between 500$1000 \mathrm{~K}$, depending on the quantity considered. For example, the calculated values of the heat capacity compare well with experiments up to approximately $1000 \mathrm{~K}$, whereas the temperature dependence of the calculated lattice parameters starts deviating from the experimental one above $1500 \mathrm{~K}$. Including the electronic contribution to the heat capacity in $\mathrm{Ru}$ (and Os) is important, as is usually the case for metals. The thermal expansion $\alpha_{1}$ is in good agreement with Arblaster's recommended values below $500 \mathrm{~K}$, but at higher temperatures the difference is significant. On the contrary, the remarkable agreement between the calculated thermal expansion $\alpha_{3}$ and Arblaster's values up to $2000 \mathrm{~K}$ is probably fortuitous since it does not correspond to a similar good agreement for $c$ and $c / a$.

It was already pointed out for Re and Tc and confirmed here for Os and Ru that the temperature variation of the $c / a$ ratio obtained from the quasi-harmonic approximation on a full $(a, c / a)$ grid is limited to $1 \%$ or less up to $2000 \mathrm{~K}$. This explains why the volume thermal expansion obtained from the full grid calculation is very similar to that obtained from the Murnaghan EOS and a constant $c / a$ with temperature.

\section{Acknowledgement}

This work was performed within the MaX Center of Excellence, with support from the European Union Horizon 2020 EINFRA program under grant agreement No 676598. Computational facilities have been provided by SISSA through its Linux Cluster and ITCS. 


\section{References}

[1] J.W. Arblaster. Johnson Matthey Technology Review, 58(3):137, 2014.

[2] D. A. Young. Phase diagrams of the elements. Univ. of California Press, 1991.

[3] Y. S. Ponosov, I. Loa, V. E. Mogilenskikh, and K. Syassen. Phys. Rev. B, 71:220301, 2005.

[4] Y. S. Ponosov, G. A. Bolotin, C. Thomsen, and M. Cardona. Phys. Stat. Sol. b, 208:257, 1998.

[5] R. Heid, L. Pintschovius, W. Reichardt, and K.-P. Bohnen. Physical Review B, 61(18):12059, 2000.

[6] H.G. Smith and N. Wakabayashi. Solid State Communications, 39(2):371, 1981.

[7] J. W. Arblaster. Platinum Metals Rev., 57(3):177, 2013.

[8] E. A. Owen and E. W. Roberts. Zeitschrift für Kristallographie, 96:497, 1937.

[9] V. A. Finkel, M. I. Palatnik, and G. P. Kovtun. Fiz. metal. metalloved., 32(1):212, 1971.

[10] R. H. Schröder, N. Schmitz-Pranghe, and R. Kohlhaas. Zeitschrift für Metallkunde, 63:12, 1972.

[11] J.W. Arblaster. Calphad, 19(3):349, 1995.

[12] J.W. Arblaster. Calphad, 19(3):339, 1995.

[13] E. O. Hall and J. F. Crangle. Acta Cryst., 10:270, 1957.

[14] R. G. Ross and W. Hume-Rothery. J. Less-Common Metals, 5:258, 1963.

[15] A.A. Tal, M.I. Katsnelson, M. Ekholm, H.J.M. JÃúnsson, L. Dubrovinsky, N. Dubrovinskaia, and I.A. Abrikosov. Physical Review B, 93(20), 2016.

[16] L. Dubrovinsky, N. Dubrovinskaia, E. Bykova, M. Bykov, V. Prakapenka, C. Prescher, K. Glazyrin, H.-P. Liermann, M. Hanfland, M. Ekholm, Q. Feng, L. V. Pourovskii, M. I. Katsnelson, J. M. Wills, and I. A. Abrikosov. Nature, 525(7568):226, 2015.

[17] X. Deng, W. Wang, D. Zhang, and K. You. Physica B: Condensed Matter, 406(3):656, 2011.

[18] C.-M. Liu, Y. Cheng, B. Zhu, and G.-F. Ji. Physica B: Condensed Matter, 406(11):2110, 2011.

[19] Y. Ma, T. Cui, L. Zhang, Y. Xie, G. Zou, J. S. Tse, X. Gao, and D. D. Klug. Phys. Rev. B, 72:174103, 2005.

[20] H. Cynn, J. E. Klepeis, C. S. Yoo, and D. A. Young. Phys. Rev. Lett., 88:135701, 2002. 
[21] K. Takemura. Phys. Rev. B, 70:012101, 2004.

[22] F. Occelli, D. L. Farber, J. Badro, C. M. Aracne, D. M. Teter, M. Hanfland, B. Canny, and B. Couzinet. Physical Review Letters, 93(9):095502, 2004.

[23] P. Souvatzis and O. Eriksson. Phys. Rev. B, 77:024110, 2008.

[24] N. Yaozhuang, X. Youqing, P. Hongjian, and L. Xiaobo. Physica B: Condensed Matter, 395(1-2):121, 2007.

[25] http://people.sissa.it/ dalcorso/pslibrary/index.html.

[26] A. Dal Corso. Computational Materials Science, 95:337, 2014.

[27] M. Palumbo and A. Dal Corso. to appear in Physica Status Solidi b, 2017.

[28] www.quantum-espresso.org/.

[29] P. Giannozzi et al. Journal of Physics: Condensed Matter, 21:395502, 2009.

[30] http://qeforge.qe-forge.org/gf/project/thermo_pw/

[31] J. P. Perdew and A. Zunger. Physical Review B, 23:5048, 1981.

[32] J. P. Perdew, K. Burke, and M. Ernzerhof. Physical Review Letters, 77:3865, 1996.

[33] J. P. Perdew, A. Ruzsinszky, G. I. Csonka, O. A. Vydrov, G. E. Scuseria, L. A. Constantin, X. Zhou, and K. Burke. Phys. Rev. Lett., 100:136406, 2008.

[34] S. Baroni, S. de Gironcoli, A. Dal Corso, and P. Giannozzi. Reviews of Modern Physics, 73:515, 2001.

[35] A. Dal Corso. Phys. Rev. B, 77:024110, 2008.

[36] S. Baroni, P. Giannozzi, and E. Isaev. Reviews in Mineralogy $\&$ Geochemistry, $71: 39,2010$.

[37] F. D. Murnaghan. Proceedings of the National Academy of Sciences of the United States of America, 30:244, 1944.

[38] D.C. Wallace. Thermodynamics of crystals. Wiley, 1972.

[39] M. Palumbo, B. Burton, A. Costa e Silva, B. Fultz, B. Grabowski, G. Grimvall, B. Hallstedt, O. Hellman, B. Lindahl, A. Schneider, P.E.A. Turchi, and W. Xiong. Physica Status Solidi (B) Basic Research, 251(1):14, 2014.

[40] G. Grimvall. Thermophysical properties of materials - enlarged and revised edition. North-Holland, Amsterdam, 1999.

[41] B. R. Sahu and L. Kleinman. Phys. Rev. B, 72:113106, 2005.

[42] X. H. Deng, W. Lu, Y.M. Hu, and H.S. Gu. Physica B, 404:1218, 2009.

[43] L. Fast, J. M. Wills, B. Johansson, and O. Eriksson. Physical Review B, 51(24):17431, 1995. 
[44] J.W. Arblaster. Calphad: Computer Coupling of Phase Diagrams and Thermochemistry, 20(3):343, 1996.

[45] F. M. Jaeger and E. Rosenbohm. Proc. Acad. Sci. Amsterdam, 34:85, 1931.

[46] V. N. Naumov, L. E. Paukov, G. Ramanauskas, and V. Ya. Chekhovskoi. Zh. Fiz. Khim., 62:25, 1988.

[47] G. R. Ramanauskas, V. D. Tarasov, V. Ya. Chekhovskoi, N. L. Korenovskii, and V. P. Polyakova. Vysokochist. Veshchestva, 4:149, 1988.

[48] J.W. Arblaster. Platinum Metals Review, 57(2):127, 2013.

[49] A. Dal Corso. Journal of Physics Condensed Matter, 25(14):145401, 2013.

[50] K. Clusius and U. Piesbergen. Z. Naturforschg., 14a:23, 1959.

[51] H. Holzmann. Siebert's Festschirft zum 50 Jahr Bestehen der Platinschmelze, page 149, 1931.

[52] A. E. Sheindlin, S. A. Kats, B. Ya. Berezin, V. Ya. Chekhovskoi, and N. L. Korenovskii. Rev. Int. Htres Temp. Refract., 12:12, 1975.

[53] E. H. P. Cordfunke and R. J. M. Konings. Thermochimica Acta, 139:99, 1989.

[54] B. Grabowski, T. Hickel, and J. Neugebauer. Physical Review B - Condensed Matter and Materials Physics, 76:024309, 2007.

[55] A. Glensk, B. Grabowski, T. Hickel, and J. Neugebauer. Physical Review Letters, 114:195901, 2015.

[56] I. Errea, M. Calandra, and F. Mauri. Physical Review B - Condensed Matter and Materials Physics, 89:064302, 2014. 